\title{
Alternanthera philoxeroides (Mart.) Griseb. (Amaranthaceae) en Navarra.
}

\section{Alternanthera philoxeroides (Mart.) Griseb. (Ama- ranthaceae) in Navarre.}

Javier Peralta de Andrés ${ }^{1 *}$

*

\section{Resumen}

Se aporta la primera cita de la especie exótica invasora Alternanthera philoxeroides en Navarra, planta con un alto potencial invasor. En la Península Ibérica se había observado con anterioridad en Galicia (A Coruña, en el año 2015), Cataluña (Barcelona, 2017) y Andalucía (Sevilla, 2021). Dado su potencial expansivo y difícil erradicación una vez extendida, convendría evaluar su distribución en Navarra para establecer medidas de control.

Palabras clave: flora vascular, especies exóticas invasoras, Península lbérica.

\begin{abstract}
This paper gives an account of the first record in Navarre of Alternanthera philoxeroides, an alien plant species with a high potential level of invasiveness. On the Iberian Peninsula, it had been observed previously in Galicia (A Coruña), in 2015, Catalonia (Barcelona, 2017) and Andalusia (Seville, 2021). Considering its capacity to spread quickly and very difficult eradication once this process has begun, it would be advisable to assess its current distribution in Navarre to implement control measures.
\end{abstract}

Key words: vascular flora, invasive alien species, Iberian Peninsula.

\section{Laburpena}

Nafarroan Alternanthera philoxeroides espezie exotiko inbaditzailearen lehen aipamena ematen da, ahalmen inbaditzaile handiko landarea. Iberiar Peninsulan orain arte ikusia zen

1 Universidad Pública de Navarra.

Departamento de Ciencias. Herbario UPNA.

Campus de Arrosadia, 31006 Pamplona-Iruña, Navarra. 
Galizian (A Coruña) 2015ean, Katalunian (Barcelona, 2017) eta Andaluzian (Sevilla, 2021). Espezie horren hedapen-ahalmen handia eta hedatu ondoren desagerraraztea zaila dela kontuan hartuta, komenigarria izango litzateke Nafarroan zer banaketa duen ebaluatzea, kontrol-neurriak ezartzeko.

Gako hitzak: flora baskularra, espezie exotiko inbaditzaile, Iberiar Peninsula.

$$
\text { r. } 2 x
$$

Alternanthera philoxeroides (Mart.) Griseb. es una planta herbácea perenne y estolonífera, de la familia Amarantáceas; tiene tallos postrados, a menudo fistulosos, hojas opuestas, sésiles y lanceoladas, y flores blancas agrupadas en glomérulos pedunculados (Clemants, 2004). Vive en medios acuáticos como hierba flotante, y también en suelos húmedos; es nativa de Sudamérica (Argentina, Brasil, Paraguay), desde donde ha llegado a otros países de América del Sur, América del Norte, Asia, Europa y Oceanía (EPPO, 2016). En Europa la primera observación se registró en 1971 en Francia, en las riberas del río Garona (Dupont, 1989) y en la actualidad se conoce del Tarn, Dordogne y Vaucluse (Farsac y Terrin, 2014).

Esta especie, de la que no se conocen citas previas en Navarra (GBIF, 2021, LordaLópez, 2013), se ha localizado en las orillas del río Arga, entre Belascoáin y Puente la Reina, en una zona con termotipo mesomediterráneo y ombrotipo subhúmedo, donde se refugian especies termófilas como Pistacia lentiscus y Smilax aspera:

ESP, Navarra, Guirguillano: río Arga. UTM 30TWN9492130092; $42.717473^{\circ} \mathrm{N}$ 1.840748 ${ }^{\circ}$ W (ETRS89), 360 m. 10/09/2021. Leg. \& Det. J. Peralta, UPNA 16950.

Se han observado dos núcleos próximos, de menos de $10 \mathrm{~m}^{2}$, con plantas flotando junto a la orilla, donde crecen Mentha aquatica, Ranunculus repens, Phragmites australis, Paspalum dilatatum y Persicaria maculosa. En el momento de la observación las plantas se encontraban en flor (Fig. 1), aunque fuera de los territorios de donde es nativa al parecer no se reproduce por semillas, sino por dispersión vegetativa, a partir de fragmentos del tallo (Cottaz et al., 2018, EPPO, 2016).

En la Península Ibérica se conoce su presencia en Galicia, donde fue detectada por primera vez en 2015, aunque es posible que estuviera instalada al menos 10 años antes (Romero y Amigo, 2015); en Cataluña fue observada en 2017 (Guardiola y Petit, 2020) y en Andalucía en 2021 (Jáuregui-Arana y García-Murillo, 2021).

Las vías de entrada de esta planta en la Unión Europea podrían ser como semilla contaminante en alimentos para aves y como plántula en plantas de vivero (EPPO, 2016); su origen en Navarra se desconoce. Romero y Amigo (2015) señalan que en la proximidad de las poblaciones de Galicia existió un vivero de plantas ornamentales y forestales; a este respecto, Guardiola y Petit (2020) mencionan un pliego de Menorca procedente de plantas de un vivero (Fernández-Rebollar, 2020). En Cataluña Guardiola 


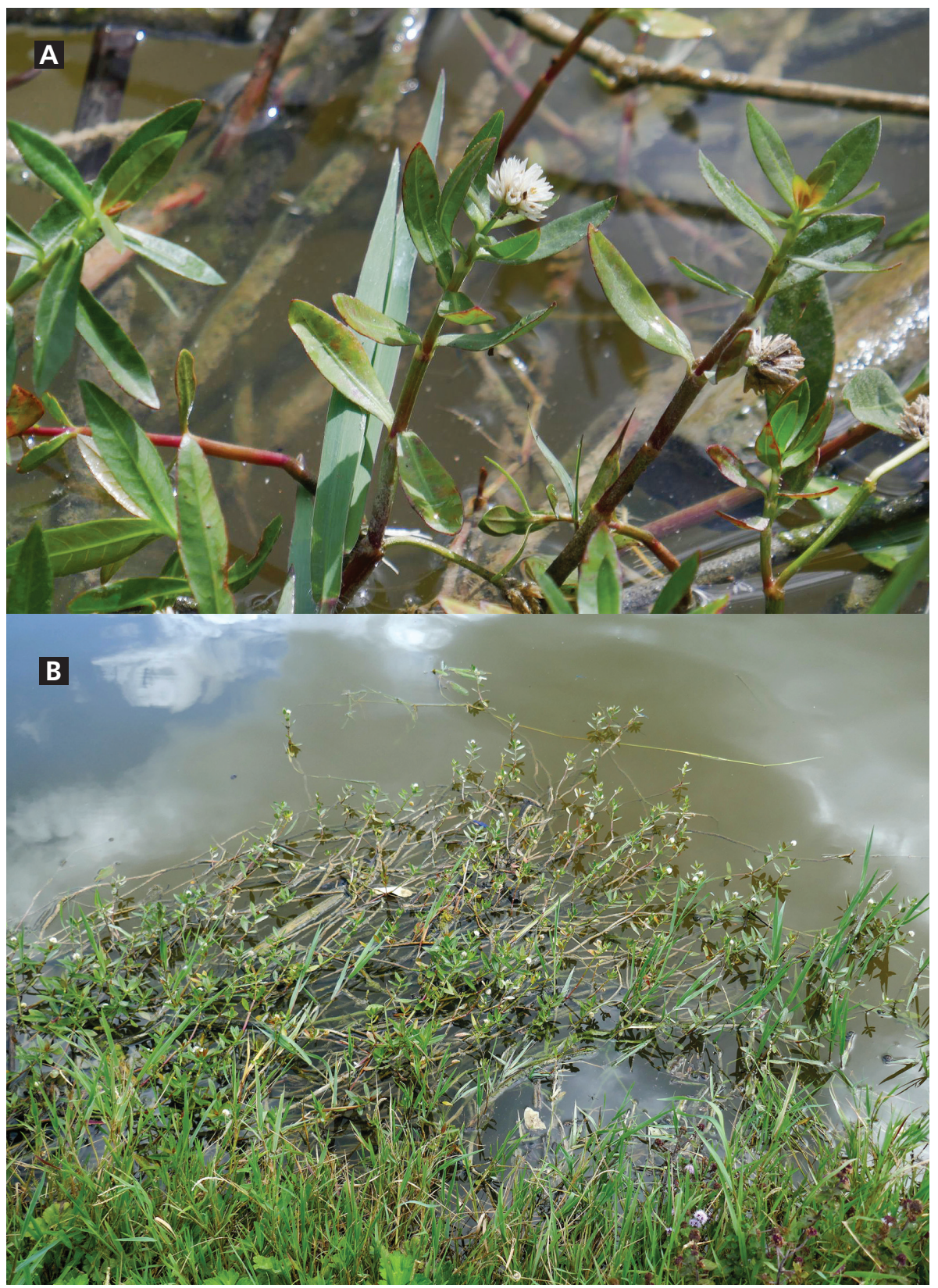

Fig.1.- (A) Althernanthera philoxeroides, hojas e inflorescencias (10.09.2021). (B) Althernanthera philoxeroides flotando junto a la orilla del río Arga (10.09.2021).

Fig. 1.- (A) Althernanthera philoxeroides, leaves and inflorescences (10.09.2021). (B) Althernanthera philoxeroides floating along the bank of river Arga (10.09.2021). 
y Petit (2020) indican su origen a través de actividades de acuicultura, como limpieza de acuarios, mientras que en Andalucía se desconoce su procedencia (Jáuregui-Arana y García-Murillo, 2021).

Es considerada una de las plantas exóticas invasoras más peligrosas por su capacidad de colonizar tanto medios acuáticos como terrestres y su difícil control (Rojas-Sandoval, 2016); en medios acuáticos podría impactar negativamente en la abundancia de otras especies de plantas, llegando a desplazarlas, y en la de macroinvertebrados (EPPO, 2016).

Esta especie figura en el "Catálogo español de especies exóticas invasoras" (BOE, 2013), donde se incluyó de modo preventivo antes de haber sido detectada en España, posiblemente por su consideración como una de las plantas acuáticas invasoras de más difícil erradicación y su presencia en Francia e Italia (CEEI, 2013). También está incluida en la "Lista de especies exóticas preocupantes para la Unión Europea" (DOUE, 2017).

En el sureste de Francia (región de Provence-Alpes-Côte d'Azur) se han ensayado métodos físicos de erradicación en medios acuáticos, con arrancado manual en años sucesivos y establecimiento de lonas permanentes, siendo este último método el más eficaz (Cottaz et al., 2018); en esta región es considerada una planta exótica invasora "emergente", con implantación reducida, y por lo tanto prioritaria para llevar a cabo acciones de gestión, ya que con medidas poco costosas es posible obtener resultados significativos como su erradicación. En España se ha modelizado el uso de control biológico de la especie mediante coleópteros (Portela et al., 2020), método de biocontrol ya utilizado en EE. UU. (Winston et al., 2017).

Dado el potencial expansivo de esta especie y su difícil erradicación una vez extendida, sería conveniente evaluar su distribución en Navarra para establecer medidas de control.

\section{Bibliografía}

BOE, 2013. Real Decreto 630/2013, de 2 de agosto, por el que se regula el Catálogo español de especies exóticas invasoras. Boletín Oficial del Estado. BOE-A-2013-8565.

CEEl, 2013. Catálogo Español de Especies Exóticas Invasoras: Alternanthera philoxeroides (Mart.) Griseb. ALTPHI/EEI/FL008. Ministerio de Agricultura, Alimentación y Medio Ambiente. www.miteco.gob.es/es/biodiversidad/temas/conservacion-de-especies/especies-exoticas-invasoras/ce_eei_flora.aspx

Clemants, S.E., 2003. Alternanthera philoxeroides in Flora of North America North of Mexico Vol. 4, 448-449. New York and Oxford. Oxford University Press. http://floranorthamerica.org/ 
Cottaz C., Paquier T., Diadema, K., 2018. L'herbe à alligator, Alternanthera philoxeroides. Expérimentation de gestion d'une espèce exotique envahissante émergente en région PACA, sur l'Ouvèze (Sorgues, 84). Conservatoire Botanique National Méditerranéen de Porquerolles.

DOUE, 2017. Reglamento de Ejecución (UE) 2017/1263 de la Comisión de 12 de julio de 2017 por el que se actualiza la lista de especies exóticas invasoras preocupantes para la Unión establecida por el Reglamento de Ejecución (UE) 2016/1141 de conformidad con el Reglamento (UE) n 1143/2014 del Parlamento Europeo y del Consejo. Diario Oficial de la Unión Europea. L 182/37.

Dupont, P., 1989. Une découverte inédite d'Emile Contré: Alternanthera philoxeroides dans la vallée de la Garonne. Bull. Soc. Bot. Centre Ouest, 20, 27-28.

EPPO, 2016. Data sheets on pests recommended for regulation - Alternanthera philoxeroides (Mart.) Griseb. Bulletin OEPP/EPPO Bulletin 46(1), 8-13.

Farsac L., Terrin E., 2014. Note d'alerte Alternanthera philoxeroides (Mart.) Griseb. Conservatoire Botanique National Méditerranéen de Porquerolles. http://www.invmed.fr

Fernández Rebollar, I., 2020. Institut Menorquí d’Estudis. Herbarium Generale Minoricae: HGM, Version 1.3. Institut Menorquí d'Estudis (IME).

https://www.gbif.org/occurrence/767640935 [acceso vía GBIF.org 31-10-2021]

GBIF, 2021. GBIF Backbone Taxonomy, GBIF Secretariat.

https://www.gbif.org/es/species/3084923 [acceso vía GBIF.org 30-10-2021]

Guardiola, M., Petit, A., 2020. Aportacions a la flora al-lòctona de la serralada Litoral central catalana i territoris propers. Butlletí de la Institució Catalana d'Història Natural 84, 35-49.

Jáuregui Arana, J., García Murillo, P., 2021. Primera cita de Alternanthera philoxeroides (Mart.) Griseb. (Amaranthaceae) en el sur de la Península Ibérica (Sevilla, España). Acta Botanica Malacitana 46. https://doi.org/10.24310/abm.v46i.11448

Lorda López, M., 2013. Catálogo Florístico de Navarra. Monografías de Botánica Ibérica 11. Ed. José Luis Benito Alonso, Jaca (Huesca).

Portela, R., Vicente, J.R., Roiloa, S.R, Cabral, J.A., 2020. A dynamic model-based framework to test the effectiveness of biocontrol targeting a new plant invader- the case of Alternanthera philoxeroides in the Iberian Peninsula. J. Environ. Manag. 264, 110349.

Rojas-Sandoval, J., 2016. Alternanthera philoxeroides. En: Invasive Species Compendium. Wallingford, UK: CAB International. www.cabi.org/isc.

Romero, M.I., Amigo, J., 2015. Alternanthera philoxeroides (Mart.) Griseb. naturalizada en el noroeste ibérico. Nova Acta Científica Compostelana (Bioloxía) 22, 1-4.

Winston, R.L., Randall, C.B., Blossey, B., Tipping, P.W., Lake, E.C., Hough-Goldstein, J., 2017. Field Guide for the Biological Control of Weeds in Eastern North America. USDA Forest Service, Forest Health Technology Enterprise Team, Morgantown, West Virginia.

Fecha de recepción/ Date of reception: 02/11/2021

Fecha de aceptación / Date of acceptance: 08/11/2021

Editor Asociado / Associate editor: Ricardo Ibáñez 